\title{
Medical Diagnostic-Based Sensor Selection
}

\author{
James B. Wendt and Miodrag Potkonjak \\ Computer Science Department \\ University of California, Los Angeles \\ \{jwendt, miodrag\}@cs.ucla.edu
}

\begin{abstract}
Wearable sensing systems have facilitated a variety of applications in Wireless Health. Due to the considerable number of sensors and their constant monitoring these systems are often expensive and power hungry. Traditional approaches to sensor selection in large multisensory arrays attempt to alleviate these issues by removing redundant sensors while maintaining overall sensor predictability. However, predicting sensors is unnecessary if ultimately the system needs only to quantify diagnostic measurements specific to the application domain. We propose a new method for optimizing the design of medical sensor systems through diagnostic-based bottom-up sensor selection. We reduce the original sensor array from ninety nine to twelve sensors while maintaining a prediction error rate of less than $5 \%$ over all diagnostic metrics in our testing dataset.
\end{abstract}

\section{INTRODUCTION}

The emergence of wireless embedded sensor networks has enabled the practical application of remote monitoring and remote control in military, wildlife, and urban environments. More recently, the development of the wearable sensing system has enabled the use of wireless embedded sensor networks to monitor the human body. This has fostered the development of devices ranging from full-bodied military suits to small, disposable medical sensors.

Current efforts aim at helping medical professionals and specialists diagnose patient illness and ailments through remote surveillance. With the assistance of wireless health sensor networks, doctors will no longer need to rely solely on the information gathered through patient interviews and onsite observations, but now have the ability to gather data from the normal day to day routine of the patient, further assisting ailment and disease diagnosis.

However, wearable sensing systems are not yet in widespread use. This is due in part to the complexity of the designs of such systems, as well as the cost of the hardware and the energy constraints that often accompany such small form wireless systems. In this paper we help to resolve these issues by reducing the size of the sensor array through diagnostic-based sensor selection. Traditional approaches to sensor selection reduce the sensor array size while maintaining overall sensor predictability [1]. However, overall sensor predictability is not necessary if the device only needs to measure diagnostic metrics specific to the medical diagnosis of interest. Thus we propose a new sensor selection methodology that optimizes the sensor array while maintaining medical diagnostic metric prediction.

\section{RELATED RESEARCH}

Embedded sensor networking and computational sensing have been applied to solve numerous scientific and engineering challenges [2] [3] [4] [5] [6] [7] [8] [9] [10] [11] $[12]$. Due to the often large networks and constant power demands of such embedded sensor systems, energy and power reduction in wireless sensor networks has been given much attention [12].

Wearable sensing systems have been attracting a significant amount of recent attention [13] [14] [15]. Growing interest in medical-based sensing devices has also fostered interest in medical-based sensing device cost and ease of use [16] [17] [18] [19] [20] [21]. While there exists energy optimization methods through hardware design, signal processing, and sensor selection [22] [23] [24] [25] [26], there is very little research applied to the cost and energy minimization of medical sensing systems specifically.

Prior research has investigated the use of gait analysis in wearable sensing systems such as sensor-equipped shoes [17] [19] [20], however, no report has yet to leverage gait analysis directly in design time sensor selection for cost reduction and energy optimization.

\section{MOTIVATION}

Individual medical sensors are often very expensive and part of larger arrays. The Hermes shoe platform used in this paper contains ninety nine sensors, and each shoe alone costs thousands of dollars. With such high costs, adoption of this system by the general public is not widespread.

While traditional sensing systems that contain large sensor arrays record very thorough measurements, they are also power hungry. Wearable sensing systems are inherently mobile devices. Frequent battery recharges, replacements, and the need to carry a large power-pack are an inconvenience for the device's users and undoubtedly deter patient use.

Our sensor selection solution alleviates these issues by reducing the size of the sensor array in medical wearable sensing systems, ultimately decreasing both the cost and power requirement. Traditional approaches to sensor selection retain full sensor predictability while removing redundant sensors [1]. However, in wireless health applications of wearable sensor systems it is often the case that we need not retain full predictability across the entire sensor network, but rather predictability of a few diagnostic metrics which correlate strongly to various diseases and ailments [27]. Thus, our approach performs sensor selection with the intent to retain only the necessary diagnostic metric predictability. 


\section{PRELIMINARIES}

\section{A. The Hermes Shoe Platform}

We evaluate our algorithm on the Hermes shoe platform, which is designed to assess balance and instability in patients [14]. It consists of ninety nine pressure sensors distributed in each insole and integrated with a common computing platform. Our sensor placement follows the Pedar plantar pressure mapping system [28] as outlined in Figure 1.

\section{B. Gait Characteristics}

VanSwearingen, et. al, [27] observe that various gait characteristics such as balance, step pressure and step stride contribute to the diagnosis of ailments and prediction of risk of falling in elderly persons. If the medical shoes assigned to a patient are ultimately to be used to help a medical professional discover these ailments or assess the risk of falling in a patient, then only these diagnostic metrics need be measured by the shoe.

We take into consideration the following diagnostic measurements which have been shown to be directly correlated to fall-frequency in the elderly: the average maximum step amplitude, the change in step stride, and the left-right pressure ratio [27].

\section{SENSOR SELECTION}

\section{A. Datasets}

Data is collected for some tens of steps, and pressure signals are recorded over all ninety nine sensors for each shoe at $60 \mathrm{~Hz}$. The resulting time-dependent pressure mappings are normalized and the aforementioned gait characteristics are extracted from the full dataset.

The processed data is separated into a training set on which to perform the sensor selection, and a testing set on which to test the sensor groupings chosen by our sensor selection algorithm.

We apply our variant of combinational iterative component assembly to the training dataset and find the best sensor groupings that predict the gait characteristics individually and collectively.

\section{B. Combinatorial iterative component assembly}

Combinatorial iterative component assembly (CICA) was first introduced in [1], however, it was originally used to find a minimal set of predictor sensors which were able to predict all other sensors to some degree of accuracy.

Similar to [1], our optimization goal is to minimize the cost and energy consumption of the sensor network by removing sensors from the original array while preserving a specified level of accuracy. CICA is a bottom-up selection process in which a subset of best sensors are chosen based on their individual prediction scores, then iteratively combined into larger groupings until a minimal set of sensors is found that collectively predicts the chosen diagnostic metrics to some specified level of accuracy. The remaining sensors from the original array are then removed. (a)

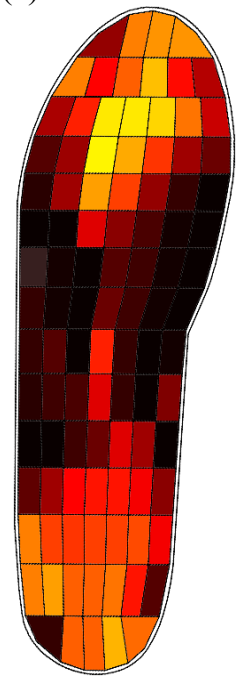

(b)

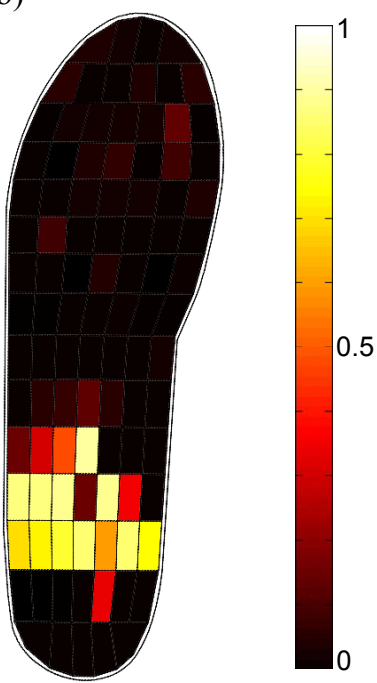

Figure 1: Individual sensor coefficient of determination of (a) average maximum step amplitude, and (b) change in step-stride. The lighter the color, the stronger the sensor acts as a predictor.

\section{CICA Algorithm}

The goal in sensor selection is to find a minimal set of sensors which can predict the gait characteristics. Our adaptation of the CICA algorithm chooses a top $k$ set of sensors based on a metric of predictability. This metric can be generated from any prediction function suited to the relationship between the sensor measurements and the gait characteristics.

In this paper we use for the measurement of prediction the coefficient of determination of the linear regression $\left(R^{2}\right.$ value $)$ between the sensor data and the observed gait characteristics. We find there is a strong linear relationship between the sensor array data and the gait characteristics, thus it is appropriate to use linear regression analysis for the CICA prediction function.

We begin by computing the individual coefficients of determination for individual sensors against each of the gait characteristics. In the first iteration we cannot compute the prediction function for each sensor for the left-right ratio gait metric since a single sensor is incapable of measuring a signal that inherently requires a minimum of two sensors to compute.

We set aside a selection set of the top $l$ individual predictors. In later iterations we combine each of the top $l$ sensors with each of the top $k$ sensor groupings to create a new set of $k$ sensor groupings with one more sensor than the previous iteration. As the iterations progress, the sensor groupings become better and better predictors of the gait characteristics.

Since we can only measure the prediction of sensors on two gait characteristics in the first iteration, we save in the selection set the top twenty five sensors that predict the stepstride metric and the top twenty five sensors that predict the average maximum amplitude. This amounts to $50 \%$ of the total number of sensors in the original array to use for sensor selection. This is more than an ample population from which to build our minimal set of sensors considering that the $R^{2}$ 
value for these sensors in our dataset ranges from near zero up to 0.90 and 0.72 for the step-stride and average maximum amplitude metrics, respectively. Since the intention is to find a minimal set of sensors that predicts all three gait characteristics, we postulate that it is highly unlikely that one of the near zero sensors will be chosen in later rounds. We find during training that this is true.

In subsequent iterations of the CICA algorithm, we combine the top $l$ sensors from the selection set with the set of $k$ sensor groupings. In the second iteration this simplifies to calculating a cross product of the selection set with itself, (a)
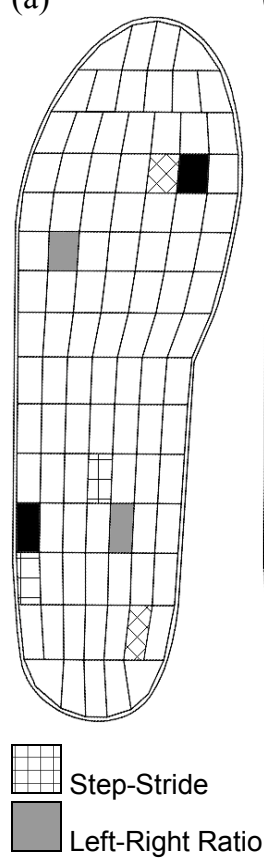

(b)

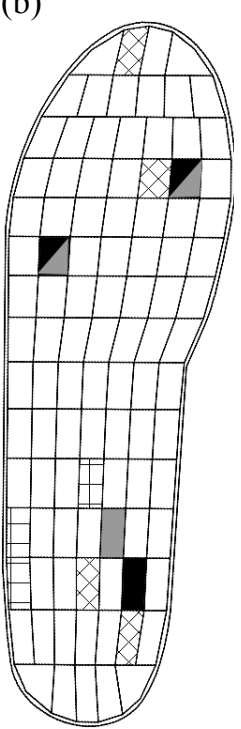

(c)

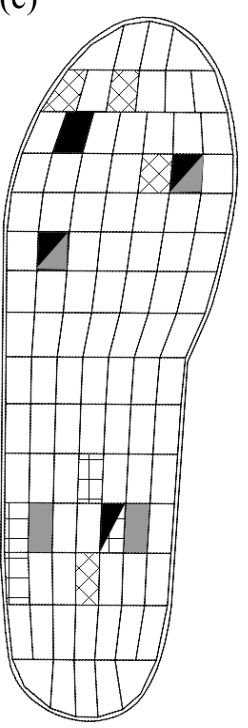

Avg Max Amplitude

\section{Overall}

Figure 2: Best sensor predictor groupings for (a) two sensors, (b) three sensors, and (c) four sensors. The Overall sensor grouping is the group of best predictors over all three diagnostic metrics found by ranking the groupings by their worst individual metric prediction values.

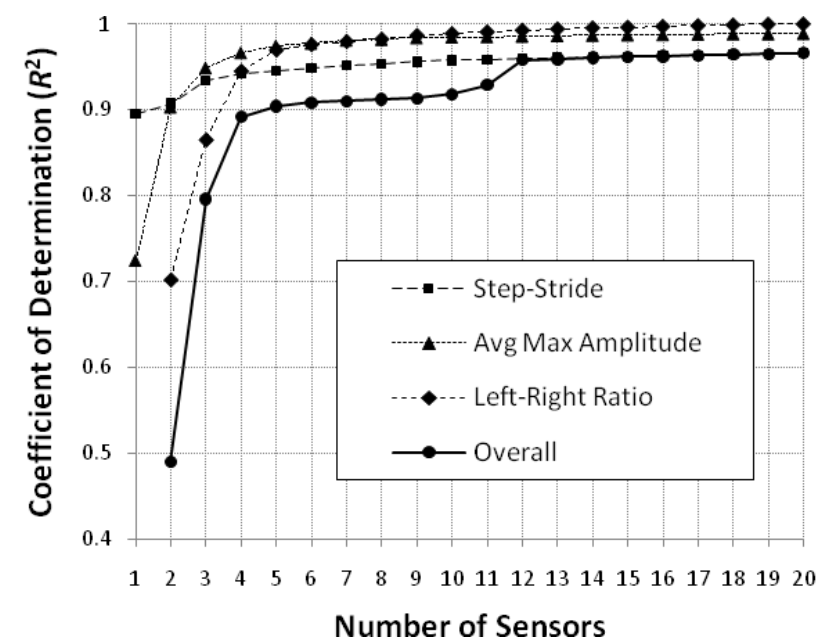

Figure 3: Predictability of best sensor groupings for each iteration. The Overall sensor groupings are determined by ranking the sensor groupings by their worst individual metric prediction values.

yielding $l^{2}$ sensor groupings of two. If we compute these cross products at each iteration the size of the sensor groupings grows exponentially, thus we limit our next iteration to only handling the top $k$ sensor groupings from the previous iteration. In this case, from the set of $l^{2}$ we take the top $k / 4$ sensor groupings that predict each of the three gait characteristics individually along with the top $k / 4$ sensor groupings ranked by their worst individual gait metric predictor. By including this fourth grouping, we help the CICA algorithm find a balance between grouping all the best sensors for a single gait metric and finding a subset of best overall sensors.

In following iterations we take the last iteration's set of $k$ sensor groupings and combine each of them with each of the $l$ individual sensors from the selection set to create a set of $k \times l$ new sensor groupings and repeat the process saving only $k$ sensor groupings for the next iteration..

The process is terminated once the minimal set of sensors that predicts the testing dataset within a specified level of accuracy is found.

\section{EXPERIMENTAL RESULTS}

A key observation in Figure $1 \mathrm{a}$ and Figure $1 \mathrm{~b}$ is that while clear correlations exist between the measurements of a few individual sensors and the average maximum step amplitude and change in step stride, between Figure 1a and Figure 1b it is not evident if there exists a single sensor that can predict both metrics. As we increase the complexity by introducing new diagnostic metrics, the task of finding the smallest subset of sensors capable of predicting all gait metrics becomes increasingly difficult.

Illustrated in Figure 2 are the best groupings of two, three, and four sensors found using our CICA variant. Because the top $k$ sensor groupings at each iteration are used in subsequent iterations, it is very possible that the top grouping changes quite dramatically from one iteration to the next, or not at all. For example, in predicting the left-right ratio metric, the best grouping from an iteration was the same as the last iteration but with an additional sensor. On the other hand, the average

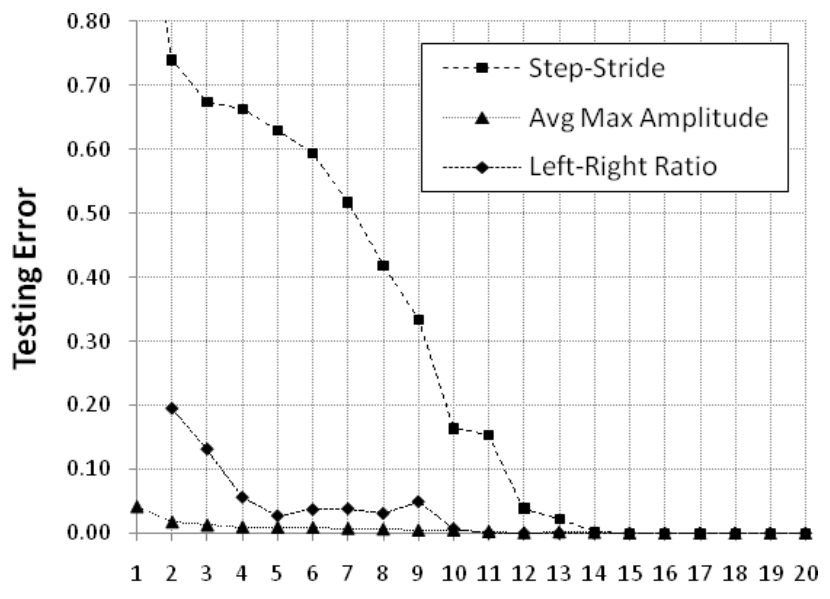

Number of Sensors

Figure 4: Applying the best sensors against the testing dataset. Testing error is measured as a percentage of actual diagnostic metric measurements. 
maximum amplitude metric saw only one sensor remain in the grouping from iteration the first iteration to the fourth. This flexibility keeps the population selection strong and diverse and helps prevent the CICA algorithm from falling into any local minimum.

We find one of the limiting factors is the predictability of the step-stride metric. While a few individual sensors had high coefficients of determination for this metric, grouping any set of sensors together did not improve its predictability as rapidly as grouping did for the other gait metrics. Figure 3 shows the average maximum amplitude and left-right ratio metrics had poor chances of predictability with small sensor groupings, but rapidly improved after a few iterations. On the other hand, the predictability of the step-stride metric remained flat even as the sensor groupings grew.

As expected, upon applying the best sensor groupings from each iteration on the testing dataset, step-stride is the worst predicted gait metric and requires a sensor grouping size of twelve before its error rate became manageable. Ultimately, we find that a minimal subset of twelve sensors is necessary to maintain a prediction error of less than $5 \%$ over all gait metrics in the testing dataset.

\section{CONCLUSION}

In this paper we explored sensor selection in medical wearable sensing systems by way of optimizing medical diagnostic metric predictability. This method aims to make expensive and power hungry wearable sensing systems more affordable and more practical for everyday use so that they may become more widespread.

By applying our variant of the CICA algorithm and optimizing for gait metric predictability, we are able to reduce the sensor array size from ninety nine sensors to twelve, a reduction of $88 \%$, while maintaining a prediction error rate of less than $5 \%$.

\section{REFERENCES}

[1] H. Noshadi, F. Dabiri, S. Meguerdichian, M. Potkonjak, M. Sarrafzadeh, "Energy optimization in wireless medical systems using physiological behaviour," Wireless Health, 2010.

[2] S. Meguerdichian, F. Koushanfar, M. Potkonjak, and M. Srivastava, "Coverage problems in wireless ad-hoc sensor networks," Infocom, vol. 3, pp. 1380-1387, 2001.

[3] S. Megerian, F. Koushanfar, M. Potkonjak, and M. B. Srivastava, "Worst and best-case coverage in sensor networks," TMC, vol.4, no. 1, pp. 84-92, 2005.

[4] S. Meguerdichian, S. Slijepcevic, V. Karayan, and M. Potkonjak, "Localized algorithms in wireless ad-hoc networks: location discovery and sensor exposure," MobiHoc, pp. 106-116, 2001.

[5] S. Slijepcevic and M. Potkonjak, "Power efficient organization of wireless sensor networks," ICC, pp. 472-476, 2001.

[6] G. Veltri, Q. Huang, G. Qu, and M. Potkonjak, "Minimal and maximal exposure path algorithms for wireless embedded sensor networks," Sen- Sys, pp. 40-50, 2003.
[7] J. L. Wong, R. Jafari, and M. Potkonjak, "Gateway placement for latency and energy efficient data aggregation," LCN, pp. 490-497, 2004.

[8] F. Koushanfar, M. Potkonjak, and A. Sangiovanni-Vincentelli, "Fault tolerance techniques in wireless ad-hoc sensor networks," Sensors, pp. 1491-1496, 2002.

[9] J. L. Wong and M. Potkonjak, "Search in sensor networks: challenges, techniques, and applications," ICASSP, vol. 4, pp. 3752-3755, 2002.

[10] J. Feng, F. Koushanfar, and M. Potkonjak, "System-architectures for sensor networks: issues, alternatives, and directions," ICCAD, pp. 112$121,2002$.

[11] J. Feng, S. Megerian, and M. Potkonjak, "Model-based calibration for sensor networks," Sensors, pp. 737-742, 2003.

[12] G. Anastasi, M. Conti, M. Di Francesco, and A. Passarella, "Energy conservation in wireless sensor networks: A survey," Ad Hoc Networks, vol. 7., no. 3, pp. 537-568, 2009.

[13] A. Krause, D. P. Siewiorek, A. Smailagic, and J. Farringdon, "Unsupervised, dynamic identification of physiological and activity context in wearable computing," ISWC, pp. 88-97, 2003.

[14] H. Noshadi, E. Giordano, H. Hagopian, G. Pau, M. Gerla, and M. Sarrafzadeh, "Remote medical monitoring through vehicular ad hoc network," VTC, pp. 1-5, 2008.

[15] K. Lorincz, B. Chen, G. W. Challen, A. R. Chowdhury, S. Patel, P. Bonato, and M. Welsh, "Mercury: a wearable sensor network platform for high-fidelity motion analysis," SenSys, pp. 183-196, 2009.

[16] S. C. Jacobsen, T. J. Petelenz, and S. C. Peterson, "Wireless health monitoring system," US Patent 6,160,478, 2000.

[17] S. Popovic, M. R. Dietz, V. Morari, M. Pappas, I. Keller, and T. Mangold, "A reliable gyroscope-based gait-phase detection sensor embedded in a shoe insole," IEEE Sensors Journal, vol. 4, no. 2, pp. 268-274, 2004.

[18] W. Wu, L. Au, B. Jordan, T. Stathopoulus, M. Batalin, W. Kaiser, A. Vahdatpour, and M. Sarrafzadeh, "The SmartCane system: an assistive device for geriatrics," Body Area Networks, 2008.

[19] K. Oshima, Y. Ishida, S. Konomi, N. Thepvilojanapong, and Y. Tobe, "A human probe for measuring walkability," Sensys, pp. 353-354, 2009.

[20] V. Erickson, A. U. Kamthe, and A. E. Cerpa, "Measuring foot pronation using RFID sensor networks," Sensys, pp. 325-326, 2009.

[21] K. Lorincz, B. Chen, G. W. Challen, A. R. Chowdhury, S. Patel, P. Bonato, and M. Welsh, "Mercury: a wearable sensor network platform for high-fidelity motion analysis," Sensys, pp. 183-196, 2009.

[22] V. Leonov, P. Fiorini, S. Sedky, T. Torfs, and C. Van Hoof, "Thermoelectric mems generators as a power supply for a body area network," vol. 1, pp. 291-294, 2005.

[23] L. Yan, L. Zhong, and N. K. Jha, "Energy comparison and optimization of wireless body-area network technologies," BodyNets, pp. 1-8, 2007.

[24] H. Ghasemzadeh, E. Guenterberg, K. Gilani, and R. Jafari, "Action coverage formulation for power optimization in body sensor networks," ASPDAC, pp. 446-451, 2008.

[25] Y. Liu, B. Veeravalli, and S. Viswanathan, "Critical-path based lowenergy scheduling algorithms for body area network systems," RTCSA, pp. 301-308, 2007.

[26] S. Xiao, A. Dhamdhere, V. Sivaraman, and A. Burdett, "Transmission power control in body area sensor networks for healthcare monitoring," IEEE Communications Journal, vol. 27, no. 1, pp. 37-48, 2009.

[27] J. M. VanSwearingen, K. A. Paschal, P. Bonino, and J. F. Yang, "The modified gait abnormality rating scale for recognizing the risk of recurrent falls in community-dwelling elderly adults", Physical Therapy, 1996.

[28] Novel.de, Pedar, 2007, http://www.novel.de/ 